Throughout the book the ground field is taken to be of characteristic zero and in fact is really intended to be the field of complex numbers.

The first seven chapters are confined to plane algebraic curves, a useful survey of the first six chapters being given at the beginning of Chapter VII. Chapter I contains a survey of the classical theory of algebraic curves. In the short Chapter II, Bezout's Theorem is introduced, the representation of point sets by two-way forms being used. In Chapter III an account is given of the four types of extension field of the ground field which are used in the book. Chapter IV deals with quasi-branches and branches. Chapter V contains the statement of Puiseaux's Theorem (proof in Appendix B). The idea of a place on a curve is introduced and results on intersection theory given in terms of places. Chapter VI deals with some traditional theory of plane algebraic curves. Chapter VII contains the theory of the function field.

Chapter VIII extends ideas and notation in the earlier chapters to curves in $S_{r}$, $r$-dimensional projective space.

Chapter IX considers curves in $S_{3}$ using the theory of Cayley forms and briefly mentions how the theory proceeds for curves in $S_{r}(r>3)$.

Chapter $\mathbf{X}$ deals with the theory of linear series on a curve. It contains a discussion of Noether's Theorem and leads up to a proof of the Riemann-Roch Theorem.

Chapter XI deals with the rather special topic of infinitely near points in the plane. This is a useful introduction to the recent generalisations in this subject.

J. HUNTER

gow, margaret M., $A$ Course in Pure Mathematics (English Universities Press, 1960), 619 pp., 40 s.

This book aims to cover the syllabus in Mathematics for Part I of the London B.Sc. General Degree, and also to provide a suitable course in Mathematics ancillary to Honours courses in Physics, Chemistry, etc.

Apart from the inadequate treatment of inequalities, Dr. Gow has achieved the the first aim. However, several important topics (Vectors, Fourier Series, Bilinear Transformations) of interest to ancillary students are omitted.

The theory of determinants is rather sketchy. Even at this level, determinants can be defined in terms of permutations and so yield the working rules.

The treatment of complex numbers is presented in great detail and well illustrated by diagrams, but on page 103 a complex number is defined as a point in the Argand diagram!

Despite a few blemishes, the treatment throughout is clear and straightforward and there are many worked examples and excellent sets of exercises.

B. SPAIN

DEFARES, J. G., AND SNEDDON, I. N., An Introduction to the Mathematics of Medicine and Biology (North Holland Publishing Co., Amsterdam, 1960), 663 pp., 94s.

This well-written book is intended to help research workers in the biological and medical sciences. Since, however, it assumes that the reader has ceased the study of mathematics for some years it can be recommended to anyone commencing his studies at an elementary level..

After the algebraic preliminaries and a discussion of functions, the differential and integral calculus is considered. There is a chapter on the logarithmic and exponential functions defined by means of integrals rather than series (on the whole, series are avoided in the book, though there is a proof of Maclaurin's theorem). A chapter on integrals, including the Gamma function, Beta function and Laplace 
transform, is followed by one on functions of more than one variable. In this the authors have managed to include an account of information theory. Another chapter is devoted to differential equations both ordinary and partial. The Laplace transform and Fourier series methods of solutions are given as well as others.

Throughout the book there are many illustrations from and applications to problems in biology and medicine, e.g. the law of allometric growth and the cupulogram in otology to select two sections at random. Every effort has been made to make the text intelligible to the student and the authors have not hesitated to advise the students not to worry about a derivation where the introduction of rigour would obscure the point of the argument. Altogether, the book should be very helpful in teaching the biologist and doctor.

D. S. JONES

\section{cunNingham, w. J., Introduction to Nonlinear Analysis (McGraw-Hill, New York, 1959), 348 pp., 70s.}

This book gives an account of numerical, graphical and analytical methods of solving ordinary non-linear differential equations. The level of presentation is determined by the fact that the book is based on a graduate course in electrical engineering given by the author at Yale. It will be of little interest to mathematicians except to provide useful illustrations to those lecturing to engineering students on differential equations.

I. N. SNEDDON

ALDER, H. L., AND ROESSLer, E. B., Introduction to Probability and Statistics (W. H. Freeman \& Co., San Francisco \& London, 1960), pp. $252+x i$, 20s.

This is intended as a textbook for a 45 -hour introductory course in probability and statistics. It is based on lectures given for several years to students in the agricultural sciences, business administration, economics, home economics, psychology, sociology, geology and the medical sciences. The only mathematical knowledge assumed in the reader is school algebra, so that students of science subjects in this country might find the pace, particularly at the beginning, rather slow. But often students who have for years lost contact with formal mathematics find a need to acquire a knowledge of statistical methods: for them the gradual introduction of this book to algebra may be very suitable.

The topics covered are organisation of data, measures of central tendency and of dispersion, elementary probability, the binomial and normal distributions, large sample methods, testing hypotheses, confidence limits, the $t$-test, the sign test, regression and correlation, the $\chi^{2}$-distribution, index numbers, and time series. The treatment of these last two topics is designed for economics students: otherwise there is no emphasis on any particular application.

For its purpose the book should be quite satisfactory. It introduces to readers of very modest attainments quite a number of standard statistical methods, well illustrated by worked examples. The exposition is clear enough, and where proofs are omitted the fact is clearly stated. There is a good selection of exercises at the end of each chapter, including " practical " ones from a variety of fields of application. Since the answers to half of them are given, the book could conceivably be used for self-study.

Such defects as have been noticed are not serious. The most striking omission is the Poisson distribution, which receives only a mention. The word "variate" is used in a sense which appears to be unusual, even in America, to denote what would more ordinarily be called a "variate-value". 